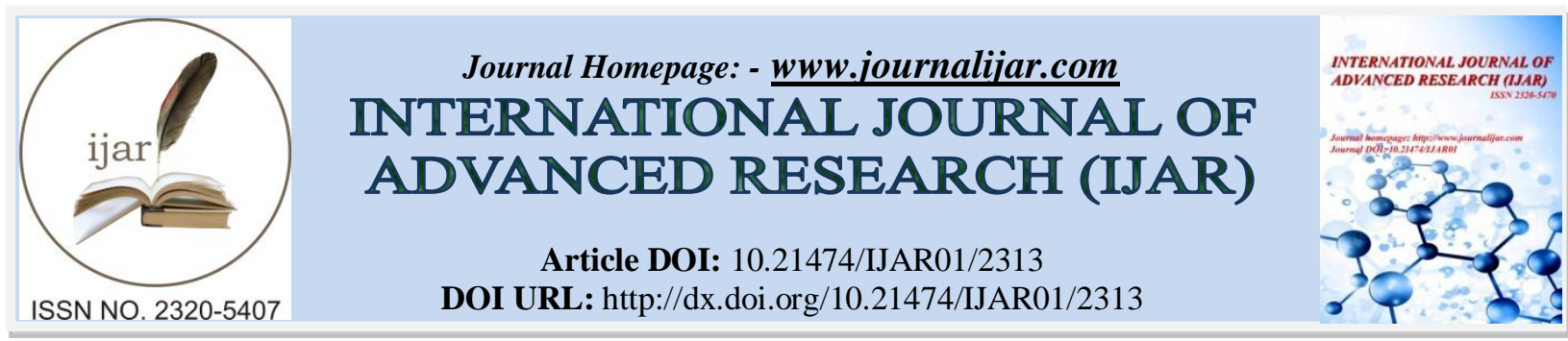

RESEARCH ARTICLE

\title{
DEVELOPMENT OF OLEOPHILIC ADSORBENT BASED ON CHITOSAN- POLY (BUTYL ACRYLATE) GRAFT COPOLYMER FOR PETROLEUM OIL SPILL REMOVAL.
}

\author{
"M. S. Mohy Eldin', Y. A. Ammar', T. M. Tamer ${ }^{3}$, A. M. Omer ${ }^{3}$ and A. A. Ali ${ }^{4}$.
}

1. Chemistry Department, Faculty of Science, University of Jeddah, Osfan, P. O. Box: 80203, Jeddah 21589, Saudi Arabia.

2. Chemistry Department, Faculty of Science, Al-Azhar University, Egypt.

3. Polymer materials research department, Advanced Technologies and New Materials Research Institute (ATNMRI), City of Scientific Research and Technological Applications (SRTA-City), New Borg El-Arab City, P.O. Box: 21934 Alexandria, Egypt.

4. Egyptian General Petroleum Corporation (EGPC), Egypt.

\section{Manuscript Info}

Manuscript History

Received: 29 September 2016

Final Accepted: 30 October 2016

Published: November 2016

Key words:-

Chitosan; poly (butyl acrylate); Graft copolymer; Hydrophobic-oleophilic characters, Oil spill removal.

\begin{abstract}
The present work was focused on the preparation, characterization and evaluation of oil adsorptive material based on chitosan- poly (butyl acrylate) graft copolymer. Chitosan (CS) was grafted with two different concentrations of n-butyl acrylate (ButA) monomer using free radical polymerization process, the prepared graft copolymers were also verified their structures, thermal stability and the morphological changes using FT-IR spectroscopy, TGA/DSC, and SEM respectively. Results showed that increasing the amount of ButA in the feed mixture enhanced the hydrophobic-oleophilic characters for the prepared grafted copolymers. Furthermore, the contact angle (wettability) measurements indicates that the grafted copolymers exhibit hydrophobic and oleophilic characters and recorded maximum values $131^{0}, 116^{0}$ compared to the native chitosan $\left(80^{\circ}\right)$ using water droplet, and $33^{\circ}, 42^{\circ}$ compared to $120^{\circ}$ for chitosan using light crude oil droplet. The selectivity of the grafted copolymers for adsorption of different oil types was studied to investigate their hydrophobic/hydrophilic and oleophilic characters. Furthermore, the evaluation of the oil spill adsorption process was also evaluated under different environmental conditions using crude oil- artificial seawater system. The increment of oil adsorption (\%) of the grafted copolymers associated also with increasing the oil viscosity in the following order; gasoil < mobil-1 oil < light crude oil < heavy crude oil. The prepared chitosan-poly (butyl acrylate) graft copolymer could be applied as a promising adsorbent for petroleum oil spill applications.
\end{abstract}

Copy Right, IJAR, 2016,. All rights reserved.

\section{Introduction:-}

Oil is the most important natural resource of the industrialized nations. It is hard to imagine any industrial operations or even private activities without oil and gas anywhere in the world. Millions of barrels of petroleum and its

Corresponding Author:- M. S. Mohy Eldin.

Address:- Chemistry Department, Faculty of Science, University of Jeddah, Osfan, P. O. Box: 80203, Jeddah 21589, Saudi Arabia. 
products are transported across the world every day. The exposure of oil spills is an alarming threat that can lead not only to the loss of valuable products, but also seriously damage the environment and ecosystems. Where, spills in shores may have economic matter in recreational areas, tourism and fisheries industry [1, 2]. Several great disaster occurred in the last 10 years, caused by ships, oil ducts and platforms that sunk or leaked or exploded, spreading large oil slick over hundreds of square kilometers [3,4]. Several common methods are used in cleanup oils spill include in situ burning of oil on water, mechanical tools (booms and skimmers), use of chemical dispersants, and synthetic sorbents $[5,6]$. The limitations of these techniques are obvious, ranging from inefficiency at high water tide, to high cost and environmental harmfulness. Among these methods, adsorption process is one of the effective methods widely used in wastewater systems. It was stated that the adsorption of oil occurs in three steps; diffusion of the oil molecules into the surface of the adsorbent materials, followed by entrapment of oil into the adsorbent material structures and finally the oil droplets agglomerated in porous and rough structures of the sorbent [7]. Furthermore, adsorbent materials such as activated carbon [8], silica aerogels [9], bentonite [10], and synthetic polymers [11] have been recorded to be one of the highly effective materials from the economic and ecologic point of view for removing oil spills on shorelines [12]. Therefore, in order to simplify the adsorption process it is necessary to develop a more efficient and environmental friendly adsorbent for oil spill cleanup. Moreover, the development of oil adsorbent made of natural organic waste materials was introduced to afford resources for marine oil spill response with less cost and environmental load [13].

Chitosan (CS) is naturally cationic polysaccharide with molar mass varied from few hundred to several million Daltons. It is produced commercially by the deacetylation of chitin which is obtained from the shells of crustaceans such as shrimps and crabs $[14,15]$. Chemically, chitosan consists of a copolymer of $\beta$ - $(1 \rightarrow 4)-2$-acetamido-2-deoxyD-glucopyranose and deacetylated unite $B$ - $(1 \rightarrow 4)$-2-amino-2-deoxy-D-glucopyranose with deacetylation greater than $60 \%$. Chitosan is characterized by its amine groups that are converted to a cationic form in acidic medium. Compared with other polymers, chitosan is becoming most popular in different applications such as medical, pharmaceutical, industrial, and water treatment applications. This is due to its excellent unique properties such as bio-degradability, biocompatibility, non-toxicity, lower density, eases modification and has lower cost as well as it has antioxidative and antimicrobial properties [16-19]. Cationic structure of chitosan simplified its use in several applications such as drug delivery, water treatment, biomedical engineering, fuel cell and food packaging [20-28]. Chitosan has been reported as a low cost alternative in the treatment of contaminated water containing phenolic compounds. The bioremediation of oil-polluted seawater has been studied using bacteria immobilized on chitin and on chitosan flakes that degrade hydrocarbons [29]. Additionally, chitosan can be chemically and physically modified for allowing the formation of functional derivatives with a highly hydrophilic and/or hydrophobic character [30].

Graft copolymerization, especially grafting of vinyl monomers onto chitosan is one of the most effective and promising method [31, 32]. Because the abundant amino groups and hydroxyl groups in chitosan backbone could react with vinyl monomers under mild conditions. So far, much work has been carried out to perform grafting copolymerization of chitosan and different vinyl monomers [33]. The main advantage in the grafting chitosan is the formation of functional derivatives by covalent binding, increasing chelating, and enhancing adsorption properties [34-36]. Modification of chitosan involving graft polymer chains has been mainly achieved via free radical polymerization, ring-opening polymerization, $\gamma$-radiation, and cationic polymerization by both grafting from and grafting to approaches [37-40]. Different studies have been published on the grafting copolymerization of chitosan with various vinyl monomers like acrylonitrile, methyl methacrylate [41], polyacrylamide, $\mathrm{N}$-isopropylacrylamide [42], and acrylic acid [40, 43].

This study focused on the development of oil adsorptive material based on low-cost commercial chitosan (CS) via grafting with hydrophobic butyl acrylate monomer (ButA) using free radical polymerization process. The grafted copolymer was characterized using different characterization tools. Additionally, the hydrophobic/hydrophilic and oleophilic characters of CS-g-poly (ButA) were evaluated through studying its selectivity for oil, water. Furthermore, the evaluation studies of the oil spill adsorption process were carried out under different environmental conditions using crude oil- artificial seawater system.

\section{Experimental:- \\ Materials:-}

Shrimp shells were collected from marine waste sources in Alexandria (Egypt). N-butyl acrylate (98\%) was obtained from Sigma-Aldrich (Germany). Potassium persulphate (KPS; 99\%) was purchased from sigma-Aldrich (Germany). Sodium hydroxide (99\%), ethanol (99\%), hydrochloric acid (purity 37\%), and acetic acid (98\%) were 
brought from El-Nasr Company (Alexandria). Engine oil (trade name Mobile-1) was supplied from Exxon Mobil Co. (Egypt). Gasoil was delivered from Misr petroleum Co. (Egypt).

Two crude oil types were used: Land Belayem (LB; heavy crude oil) and Marine Belayem (MB; light crude oil) were supplied from Belayem Petroleum Company, (Egypt). Furthermore, density and viscosity values of the used oil types were investigated in Table 1.

Table 1:- Measurement values of both density and viscosity for the used oil types

\begin{tabular}{|c|c|c|}
\hline Type of oil & Density $\left(\mathrm{g} / \mathrm{cm}^{3}\right)$ & Viscosity (Sec.) \\
\hline Gasoil & 0.790 & 30 \\
\hline Mobil-1 oil & 0.856 & 48.5 \\
\hline Light crude oil & 0.8923 & 70 \\
\hline Heavy crude oil & 0.9249 & 490 \\
\hline
\end{tabular}

\section{Methods:-}

\section{Extraction of chitin:-}

The de-mineralization of shrimp shells is the main process for chitin preparation [44]. Briefly, the shells were scattered in 5\% (w/v) $\mathrm{HCl}$ at ambient temperature in the ratio of 1:14 (w/v) and left overnight. Then, the shells were rinsed using water to remove acid and calcium chloride. The de-mineralized shells were treated with 5\% (w/v) $\mathrm{NaOH}$ at room temperature for $24 \mathrm{hr}$ in the ratio of $12: 1(\mathrm{v} / \mathrm{w})$. The residues were collected and washed to neutrality many times in running tap water and then; distilled water to obtain pure chitin.

\section{Preparation of chitosan from chitin:-}

Chitosan (CS) was prepared via deacetylation process of chitin in alkaline medium [45]. Where, the removal of acetyl groups from chitin was achieved using $50 \%(\mathrm{w} / \mathrm{v}) \mathrm{NaOH}$ with a solid to solution ratio of 1:50 (w/v) at 100$120^{\circ} \mathrm{C}$ for $12 \mathrm{hr}$. The produced chitosan was washed several times with distilled water, and left to dry at $40^{\circ} \mathrm{C}$. Finally, the dried chitosan was purified by dissolving a desired amount of CS in $2 \%(\mathrm{w} / \mathrm{v})$ acetic acid and left overnight. Then, the solution was filtrated to remove the un-dissolved particles and followed by precipitation with $5 \%(\mathrm{w} / \mathrm{v}) \mathrm{NaOH}$, collected and washed again with distilled water to remove the excess of $\mathrm{NaOH}$.

\section{Preparation of chitosan- poly (butyl acrylate) graft copolymer:-}

Chitosan-poly (butyl acrylate) graft copolymer was prepared as following; $0.2 \mathrm{~g}$ of chitosan was dissolved in $20 \mathrm{ml}$ acetic acid solution (2\%) at room temperature, then $10 \mathrm{ml}$ of ethanol was add drop wise to solution under vigorous stirring. $0.1 \mathrm{~g}$ of potassium persulphate (KPS) was dissolved in $50 \mathrm{ml}$ distilled water and added to the chitosan solution, the reaction temperature was raised to $60^{\circ} \mathrm{C}$. After $20 \mathrm{~min}$, butyl acrylate $(10$ and $20 \mathrm{ml})$ was injected drop by drop in simultaneous with additional second portion of KPS $(0.05 \mathrm{~g}$ dissolved in $5 \mathrm{ml})$. The grafting reaction was conducted for $3 \mathrm{hr}$. The precipitated products were recovered by centrifugation. To eliminate the butyl acrylate homopolymer from the graft copolymer, the resultant precipitate was washed several times with acetone and pure methanol using soxhlet for $2 \mathrm{hr}$, and then dried at $50^{\circ} \mathrm{C}$. The resultant graft copolymers were coded as CS-g-poly (ButA 10) and CS-g-poly (ButA 20). Schematic diagram represented the proposed mechanistic pathway for synthesis of CS-g-poly (ButA) is presented in Fig. 1.

Both grafting percent (Gp \%) and grafting efficiency (GE \%) were calculated as follows [46, 47]:

$\mathrm{GP}(\%)=((\mathrm{W} 1-\mathrm{W} 0) / \mathrm{W} 0) \times 100$

$\mathrm{GE}(\%)=((\mathrm{W} 1-\mathrm{W} 0) / \mathrm{W} 2) \times 100$

Where, $\mathrm{W}_{0}$ is weight of chitosan, $\mathrm{W}_{1}$ is weight of chitosan-poly (ButA) graft copolymer, and $\mathrm{W}_{2}$ is the weight of ButA monomer. 

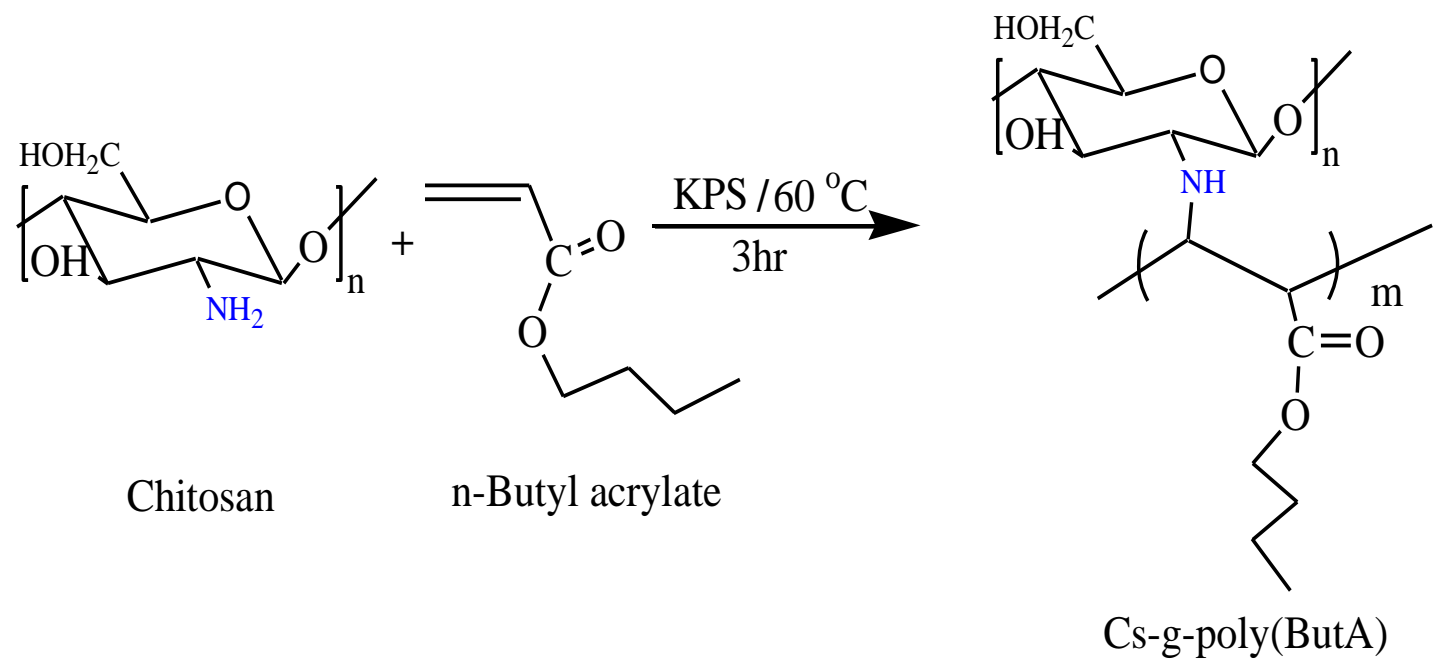

Fig. 1:- Synthesis of chitosan- poly (butyl acrylate) graft copolymer.

\section{Physicochemical characterization:-}

Infrared spectroscopic analysis:-

The chemical structures of the chitosan and chitosan- poly (butyl acrylate) graft copolymer were investigated by using Fourier Transform Infrared Spectrophotometer (Shimadzu FTIR - 8400 S, Japan).

\section{Thermal properties:-}

Thermal stability of chitosan and its grafted copolymers were estimated using Thermal gravimetric Analyzer (Shimadzu TGA -50, Japan) and Differential scanning calorimeter (Shimadzu DSC-60-A, Japan).

\section{Scanning electron microscope (SEM)}

The changes in the surface morphology of chitosan and its grafted copolymer were observed with the help of a scanning electron microscope (SEM; Joel Jsm 6360LA, Japan).

\section{Contact angle measurement:-}

The hydrophobic/hydrophilic characters of chitosan and the prepared graft copolymers were investigated by using contact angle measurement (Rame-hart instrument Co. Model 500-F1, UK) using light crude oil and distilled water droplets.

\section{Water/oil uptake measurements:-}

Briefly, the uptake behavior or both chitosan and the prepared grafted copolymers was measured by immersing $0.1 \mathrm{~g}$ of each sample in $25 \mathrm{ml}$ of liquid (water, Gasoil, Mobil-1 oil, Light crude oil, and Heavy crude oil) under constant shaking rate $(100 \mathrm{rpm})$ for a definite time $(2 \mathrm{hr})$. The samples were separated gently and the excess of adherent liquid was removed using filter paper, then weighed immediately in a closed electronic balance.

The liquid uptake (LU) can be expressed by the following equation:

$\mathbf{L U}(\%)=((\mathbf{W s}-\mathbf{W O}) / \mathbf{W 0}) \times \mathbf{1 0 0}$

Where; Ws is the weight of swollen sample, and $\mathrm{W}_{0}$ is the initial dry weight of sample.

\section{Batch oil adsorption experiments:-}

Batch adsorption studies were performed based on the Standard Test Method for adsorbent performance of adsorbent materials (ASTM F726-99) [48] using oil-artificial sea water system with salinity 3.5\% NaCl. Different amounts of oil $(2.5-15 \mathrm{~g})$ were poured into a $500 \mathrm{ml}$ beaker containing $300 \mathrm{ml}$ of artificial sea water, then different amount of adsorbent samples $(0.1-1 \mathrm{~g})$ were dispersed on the oil-water surface under different shaking rates (50-200 $\mathrm{rpm})$ at different adsorption temperatures $\left(25-40^{\circ} \mathrm{C}\right)$ for a definite contact time period (10-300 $\left.\mathrm{min}\right)$. The used oil types were gasoil, mobil-1 oil, light and heavy crude oil. Finally, samples were removed and weighted using electronic balance. The oil adsorption (\%) was measured by the following equations [49, 50 ]:

Oil adsorption $(\%)=(\mathbf{W a} / \mathbf{W b}) \times 100$

Where; Wa is the weight of adsorbed oil (g), and $\mathrm{Wb}$ is the initial weight of oil (g). 


\section{Results and Discussion:-}

Indeed, Grafting of synthetic acrylate monomers on to natural polymers surface via a free radical polymerization technique produces radicals in specific sites contributing to the grafting process. The soluble initiator (KPS) dissolves in the chitosan solution and the and start initiation step forming $\cdot \mathrm{SO}_{4}{ }^{-}$radicals which attack the amino groups and produce active sites on the chitosan macromolecules chains, and subsequently reacting with the $\mathrm{n}$-butyl acrylate monomers forming poly butyl acrylate grafted chitosan copolymer.

The effect of n-butyl acrylate concentration on the grafting percentage (GP\%) and grafting efficiency (GE\%) was shown in Fig. 2. It was clear from results that the grafting percentage increased from 71.6 to $91.3 \%$ with increasing the amount of n-butyl acrylate. Increasing the monomer concentration facilates the diffusibility of monomer towards the initiated sites on the chains of chitosan backbone, also this could be ascribed to the substantial amount of poly (ButA) grafted on the substrate backbone (CS) and this consequently increases the grafting percentage. However, grafting efficiency displays different trend than grafting percentage, increasing the concentration of ButA monomers from 10 to $20 \mathrm{ml}$ decreases the GE\% could be related to increasing the divisor value. Although, both GP\% and GE\% exhibited to be exceed than $65 \%$ for the two concentrations used.

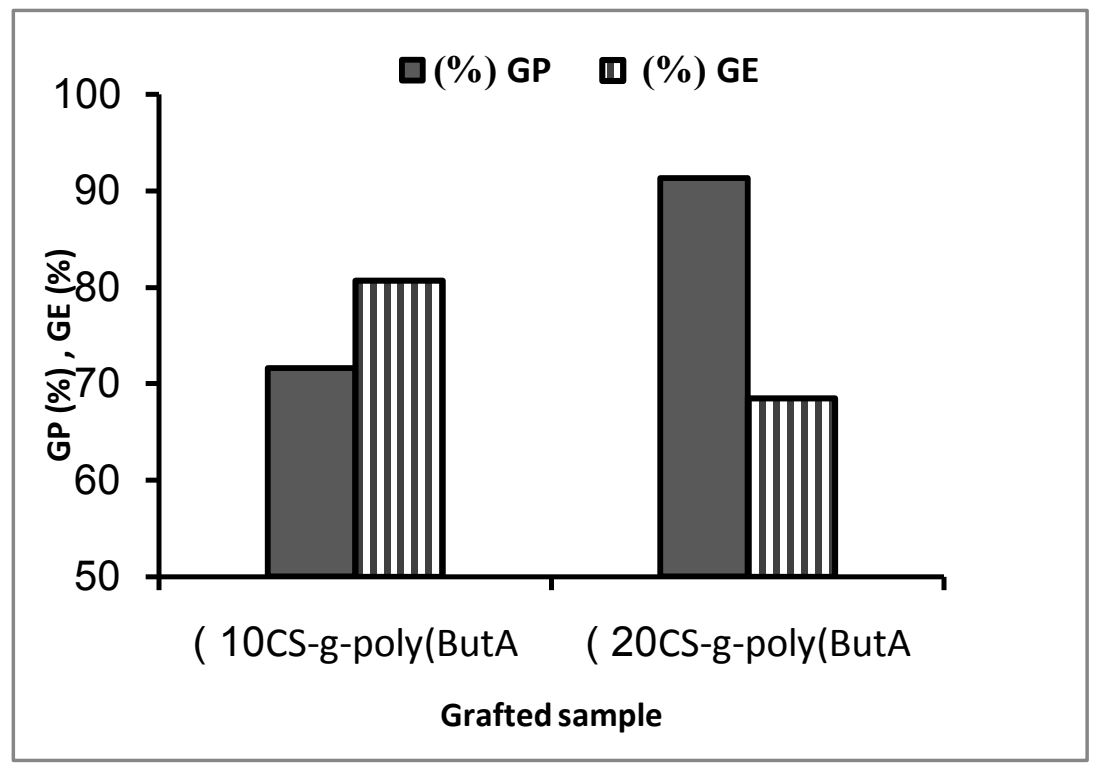

Fig. 2:- Grafting percentage (GP\%) and grafting efficiency (GE\%) values for the prepared grafted copolymers.

\section{FTIR spectroscopy:-}

Fig. 3 represents FTIR of Chitosan, Cs-g-poly (ButA10) and Cs-g-poly (ButA20). Chitosan demonstrates the typical bands of chitosan function groups; a broad band between $3200-3600 \mathrm{~cm}^{-1}$ matching to the stretching vibration of $\mathrm{NH}_{2}$ and $\mathrm{OH}$ groups that distributed along the polymer backbone. Bands between $2835-2950 \mathrm{~cm}^{-1}$ in a combination of C-H stretching of methyl and methylene groups, Bands at $1620 \mathrm{~cm}^{-1}$ result stretching vibration of $\mathrm{C}=\mathrm{O}$ and $\mathrm{NH}-$ $\mathrm{C}=\mathrm{O}$. Bands at 1066-1059 $\mathrm{cm}^{-1}$ corresponding to $\mathrm{C}-\mathrm{O}-\mathrm{H}$ stretching. Grafting of poly (butyl acrylate) into chitosan shows a significant change in FT-IR Spectra. Sharp absorption bands of the $\mathrm{C}=\mathrm{O}$ group stretching vibration has appeared at $1729 \mathrm{~cm}^{-1}$, and the typical absorption bands of the $\mathrm{C}-\mathrm{H}$ stretching vibration and the $\mathrm{C}-\mathrm{H}$ in-plane bending vibration looked at $2876,2930 \mathrm{~cm}^{-1}$ and $1380,1470 \mathrm{~cm}^{-1}$, respectively. 


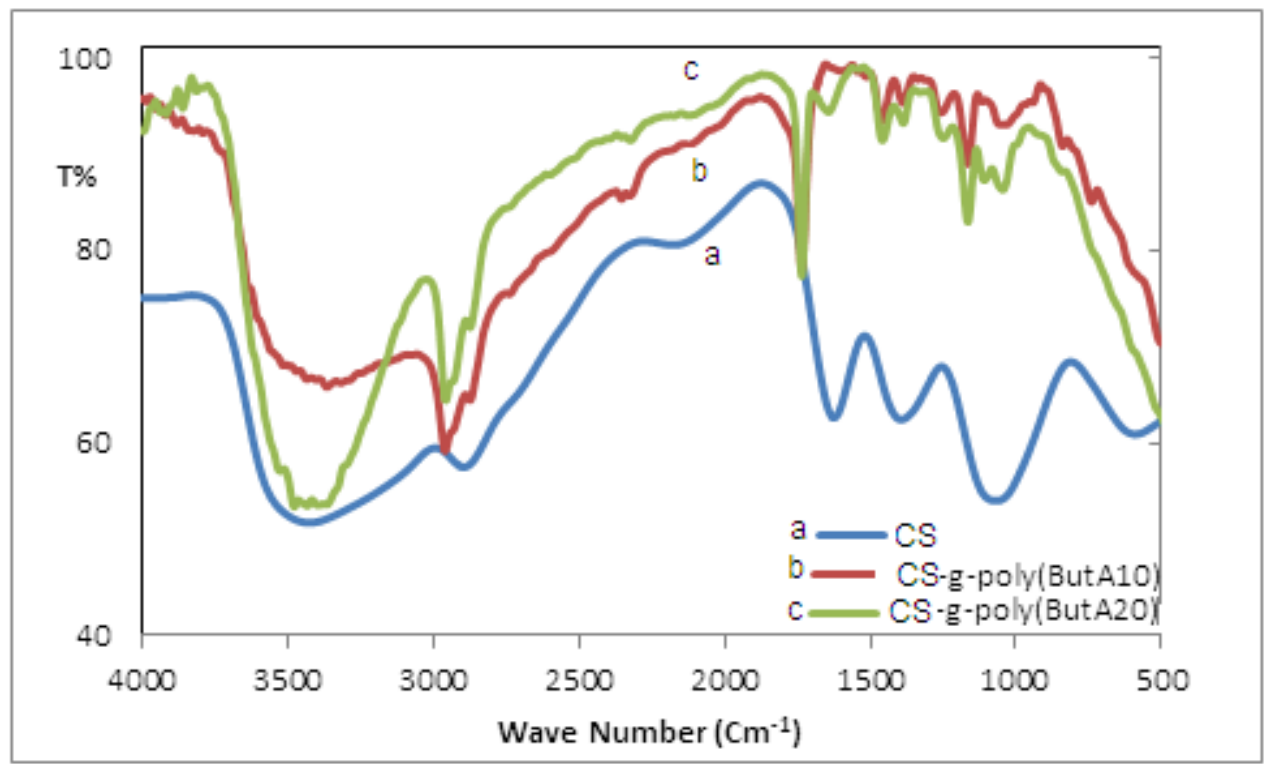

Fig. 3:- FT-IR spectra of CS, CS-g-poly (ButA 10), and CS-g-poly (ButA 20) copolymers.

\section{TGA}

Fig. 4 and Table 2 represent the thermal gravimetric analysis of CS, CS-g-poly (ButA 10) and CS-g-poly (ButA 20). Chitosan demonstrate three weight loss stops. The first weight loss that starts from ambient temperature to about $150^{\circ} \mathrm{C}$ was related to elevate moisture molecules that interrupted in polymer chain. Presence of hydrophilic groups (hydroxyl and amine groups) along polymer backbone can be explaining the ability of polymer to trap moisture from surround atmosphere.

Table 2:- Thermal gravimetric parameters of CS, CS-g-poly (ButA 10), and CS-g-poly (ButA 20)

\begin{tabular}{|c|c|c|}
\hline Sample & $\begin{array}{c}\text { Weight loss }(\boldsymbol{\%}) \\
\text { ambient }-\mathbf{1 5 0}^{\mathbf{}} \mathbf{C}\end{array}$ & $\mathbf{T}_{\mathbf{5 0}}\left({ }^{\circ} \mathbf{C}\right)$ \\
\hline Chitosan & 11.16 & 346.85 \\
\hline CS-g-poly (ButA 10) & 9.23 & 386.37 \\
\hline CS-g-poly (ButA 20) & 1.87 & 384.37 \\
\hline
\end{tabular}

The subsequent degradation that identified from $220^{\circ} \mathrm{C}$ to $320^{\circ} \mathrm{C}$ was a result of thermal oxidative decomposition of the pyranose ring along chitosan backbone. In this stage, the depression was generated from the destruction of amine groups to form crosslinked residue [51]. The third degradation step revels to the decomposition of a new crosslinked residue produced by thermal crosslinking reactions at higher temperature [52].

CS-g-poly (ButA 10) and CS-g-poly (ButA 20) copolymers demonstrate different decomposition behavior. Both derivatives showing less moisture content $9.23 \%$ and $1.87 \%$ respectively, this could be explained by the hydrophobic nature of poly (butyl) acrylate. Poly (butyl acrylate) demonstrates a single degradation step between $380-400{ }^{\circ} \mathrm{C}$ [53]. The obtained results indicate that the grafted chitosan-poly (ButA) copolymers have higher thermal stability than chitosan native polymer. 


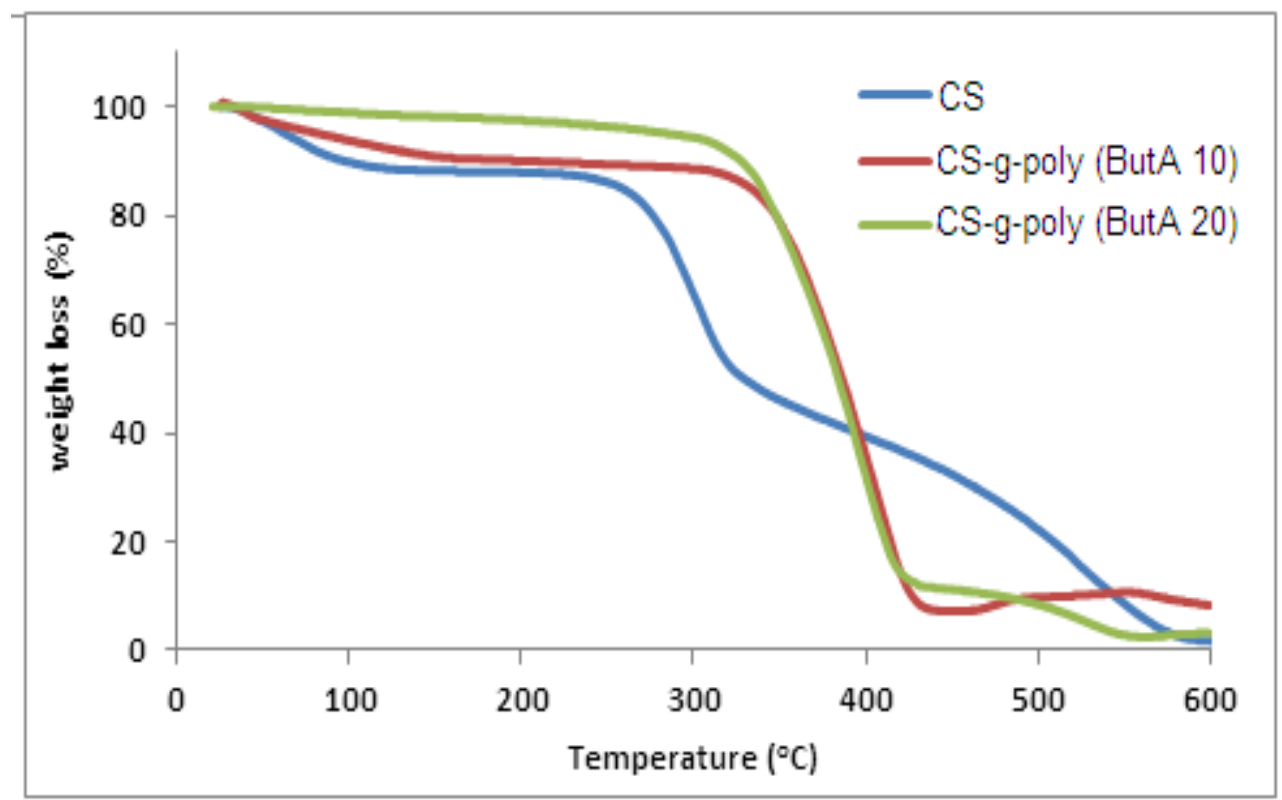

DSC

Fig. 4:- TGA of CS, CS-g-poly (ButA 10), and CS-g-poly (ButA 20) copolymers.

Fig. 5 presented differential scanning calorimetry of CS, CS-g-poly (ButA 10), and CS-g-poly (ButA 20). The results demonstrate a clear endothermic band around $100^{\circ} \mathrm{C}$ that assigned to dehydration of moisture content of samples, Chitosan as all polysaccharide has hydrophilic groups such as hydroxyl and amine groups, the ability of these groups to hold molecules of water from surrounding or during preparation increase with rising the hydrophilic nature of polymer backbone. The relatively large amount of binding water in chitosan was decreased in chitosan grafted derivative under the influence of hydrophobic nature of poly (butyl acrylate).

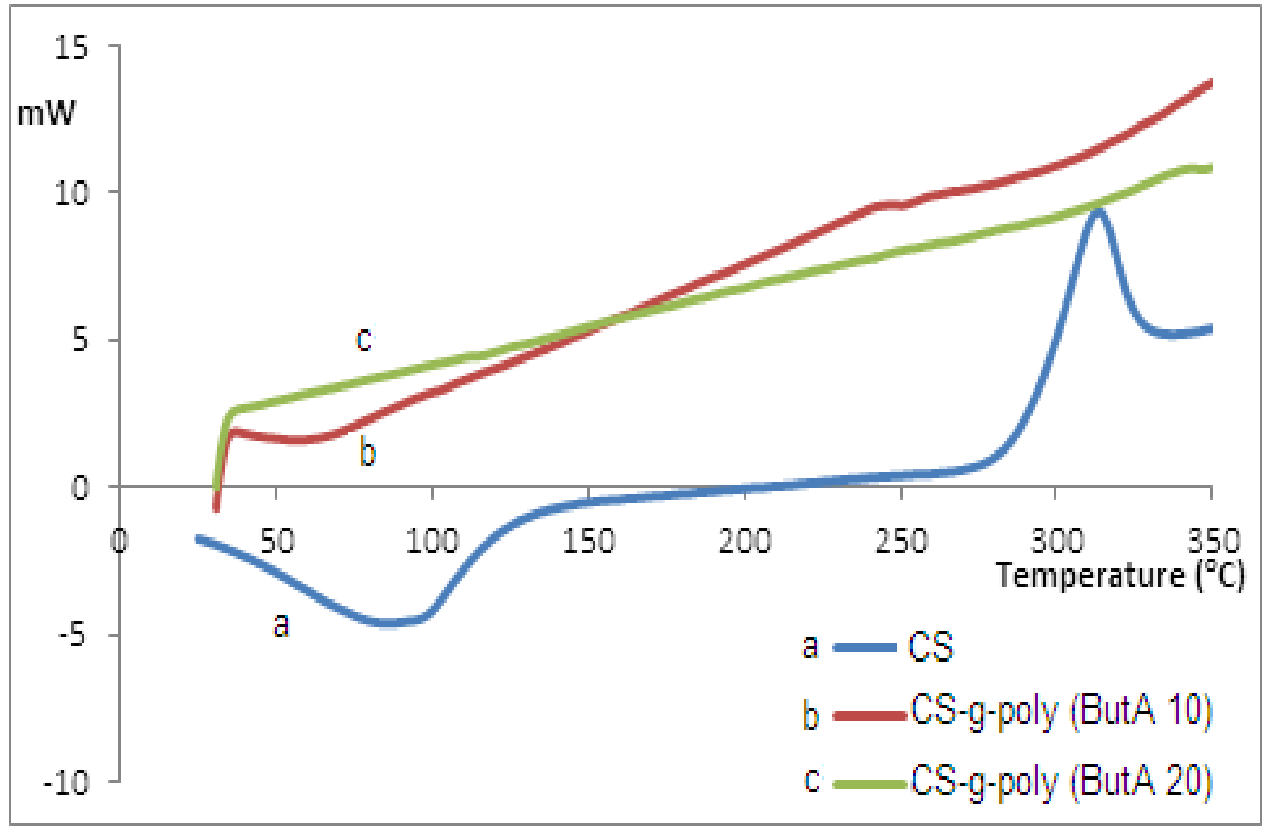

\section{SEM}

Fig. 5:- DSC of CS, CS-g-poly (ButA 10), and CS-g-poly (ButA 20) copolymers.

Scanning electron microscope represents the morphological structure of chitosan, and its derivatives as shown in Fig. 6. It was clear from SEM images that the grafted copolymers demonstrate a smooth homogenous structure compared to the native chitosan structure. This behavior can be attributed to the resultant hydrophobic nature of the prepared graft copolymers due to presence of the hydrophobic poly (butyl acrylate). 


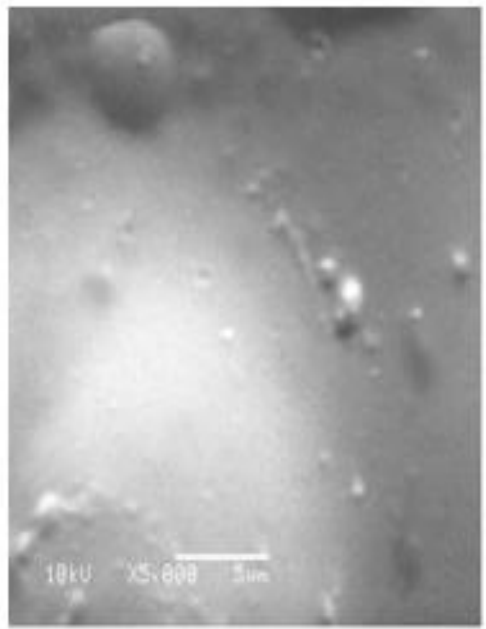

CS

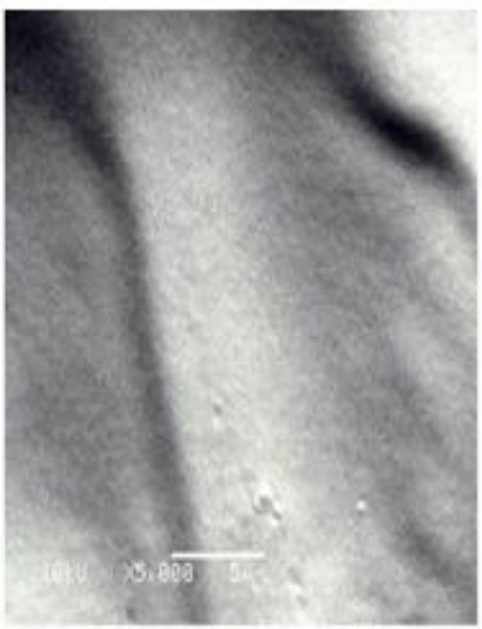

CS-g-poly (ButA 10)

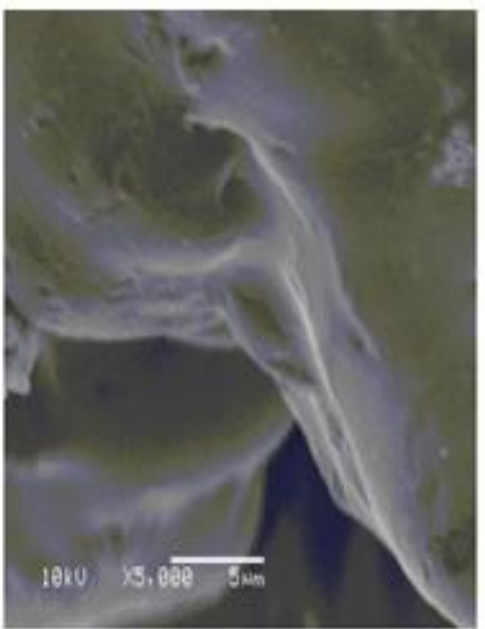

CS-g-poly (ButA 20)

Fig. 6:- SEM images of Cs, CS-g-poly (ButA 10), and CS-g-poly (ButA 20) copolymers.

\section{Contact angle}

Contact angle measurements are used to investigate the hydrophobic/hydrophilic and oleophilic characters of chitosan and the prepared graft copolymers using light crude oil and water droplets. It is very important in understanding the wetting and adhesion properties that are essential for the sorbent materials that used in oil spill cleanup. According to Fig. 7 and Table 2, it was observed that the hydrophobic and oleophilic characters of the grafted copolymers were greatly improved and reached maximum values $\left(116^{\circ}, 42^{\circ}\right)$ and $\left(131^{\circ}, 33^{\circ}\right)$ respectively for CS-g-poly (ButA 10 ) and CS-g-poly (ButA 20) compared to the native chitosan, that recorded $\left(80^{\circ}\right.$ and $120^{\circ}$ ) using water and light crude oil droplets. These results could be attributed to the hydrophobic and oleophilic nature of the used ButA monomers which increases with increasing its amount in the samples used.

Table 2:- Data of contact angle for chitosan and the grafted copolymers using water and light crude oil droplets

\begin{tabular}{|c|c|c|}
\hline $\begin{array}{c}\text { Sample } \\
\text { code }\end{array}$ & $\begin{array}{c}\boldsymbol{\theta} \\
\text { (Water) }\end{array}$ & $\begin{array}{c}\boldsymbol{\theta} \\
\text { (Light crude oil) }\end{array}$ \\
\hline CS & 80 & 120 \\
\hline CS-g-poly(ButA10) & 116 & 42 \\
\hline CS-g-poly(ButA 20) & 131 & 33 \\
\hline
\end{tabular}

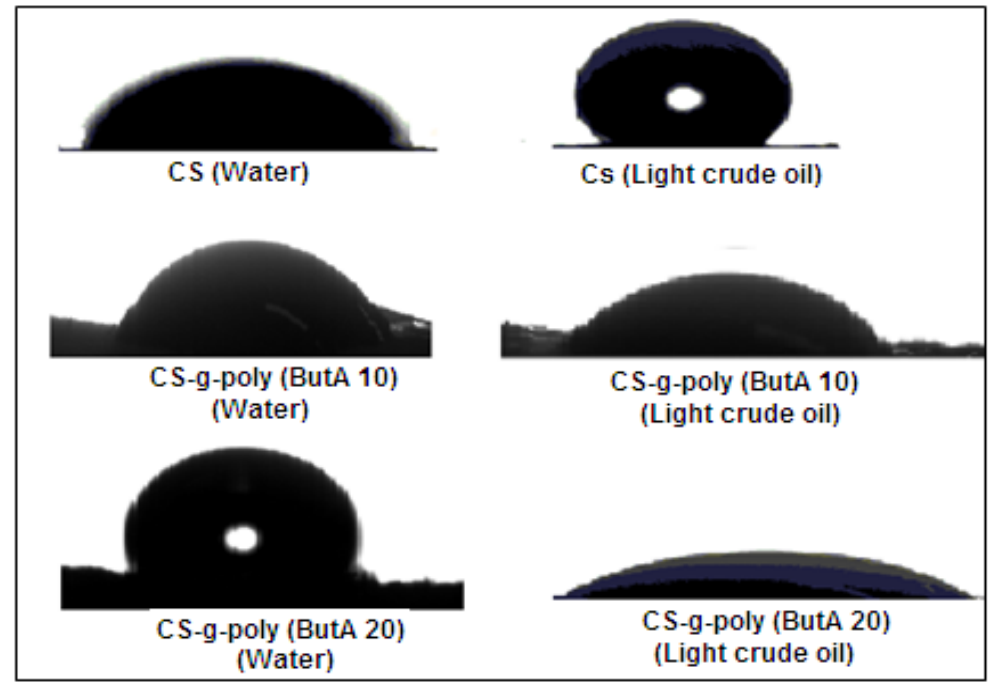

Fig. 7:- Contact angle images for chitosan, CS-g-poly (ButA 10), and CS-g-poly (ButA 20) using water and light crude oil droplets. 


\section{Water/oil uptake behaviors:-}

Water and different oil uptakes of the chitosan, CS-g-poly (ButA 10), and CS-g-poly (ButA 20) were investigated as shown in Fig. 8. The obtained results display a remarkable increase in water uptake of chitosan compared to the grafted and this increment can be attributed to the higher hydrophilicity of the native chitosan. Where, the hydrophilic nature of chitosan was assigned to the hydrophilic groups (i.e.; hydroxyl and amine groups) that spread along chain backbone which support polymer hydrophilicity. However, a sharp decline in the water uptake values was observed with increasing the amount of ButA monomer in the feed mixture during the grafting process, this can be explained by increasing the hydrophobic nature of the resultant graft copolymers with increasing the grafted number of ButA molecules on the surface of chitosan polymer.

On the other hand, oil uptake increased gradually with increasing the grafted amount of ButA monomer on the chitosan backbone. It is important to note that the changes in the oil uptake values may predominantly depend on the density and the viscosity of the oil used. Where, oil uptake amount increased with increasing the viscosity of oil used. The presence of hydrocarbon long chains of butyl acrylate monomers in addition to the decrease in the free hydrophilic amine groups as well as increasing the hydrophobic character of the grafted copolymer enhances the oil sorption process and then increasing its oil uptake values consequently.

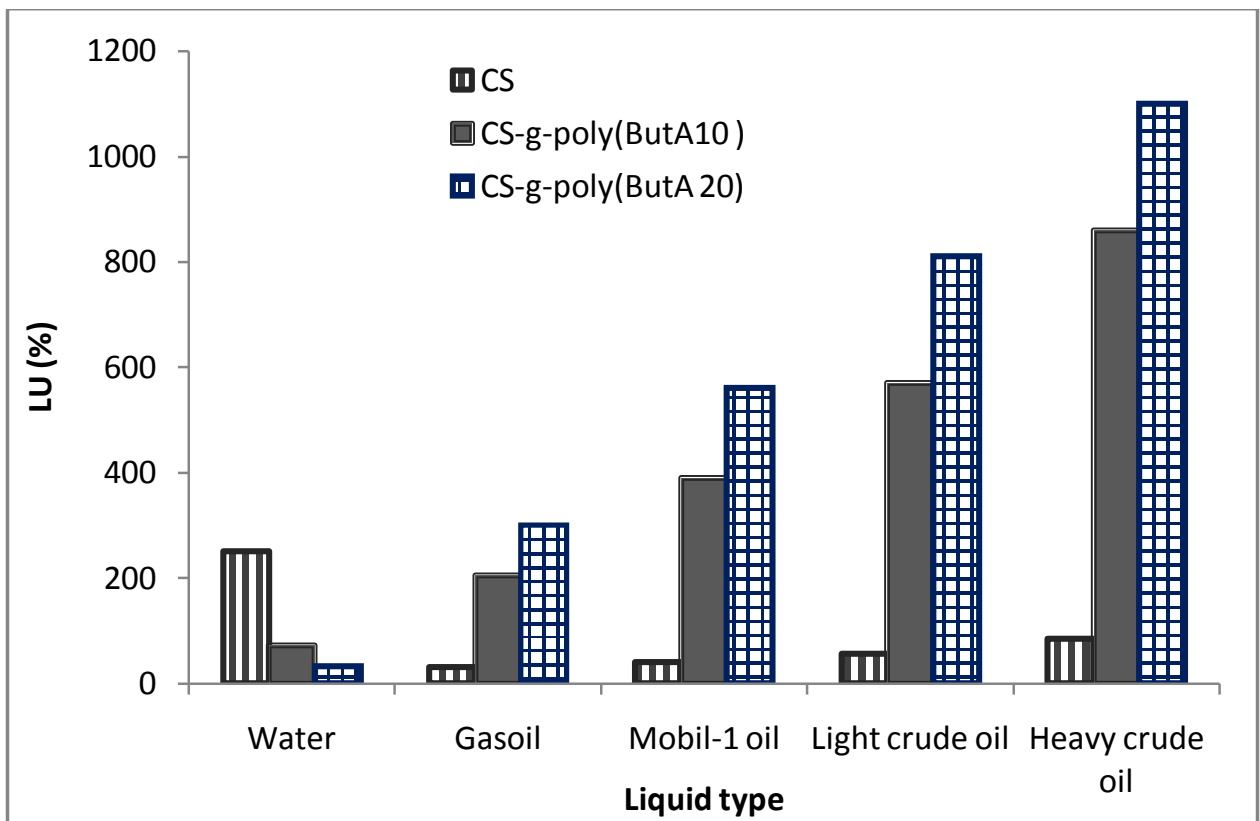

Fig. 8:- Liquid uptake behaviors of CS, CS-g-poly (ButA 10), and CS-g-poly (ButA 20) in water and different oil

\section{Oil adsorption studies:-}

types.

In fact, when oil is poured over the sea water surface, it spreads as much as possible and it tends to stick to any solid surface. Indeed, the oil adsorption process of the cydrophilic chitosan polymer is not yet fully understood, howeverbut some reports stated two different adsorption mechanisms for chitosan based on experimental clarifications [54]: (i) chitosan can acts as a network, getting involved around the oil drops and contact them (i.e Tangle effect), and (ii) the oposite charges in the oil and chitosan molecules can attarct each other producing the adsorption process.

On the other hand the hydrophobic-oleophilic nature of butyl acrylate reflects positively on the prepared chitosan grafted poly (butyl acrylate) copolymer, where the adsorption process was enhanced after grafting process and this was also confirmed previously by oil uptake studies (Section 3.6). Fundamentally, chitosan and the grafted copolymers were evaluated their affinity for oil adsorption under simulation of the sea water conditions as following:

Effect of oil type:-

Four oil types with different viscosity values were used for studying the effect of oil type on the oil adsorption percentage for chitosan and the grafted copolymers as shown in Fig.9a. The experiment was conducted under the 
simulated conditions of sea water surface. It was noticed that the oil adsorption percentage was increased gradually with increasing oil viscosity, where the adsorption values increased using oil with the following order; Gasoil < Mobil-1 oil < Light crude oil < Heavy crude oil. Where, maximum values for adsorption were recorded using the highest viscous one (i.e. heavy crude oil). These results can be attributed to with increasing oil viscosity the adherent forces between oil surface and the adsorbent materials increase and so the adsorbed amount of oil increases consequently. Fundamentally, high oil viscosity can induce adsorption properties by enhancing the adherence of oil onto the surface of the adsorbent material. On the other hand, it was observed that the adsorption values of the grafted copolymers were much high (78\%) compared to the native chitosan (33\%) using heavy crude oil, also increasing the amount of butyl acrylate used enhanced the oil adsorption process. These observations could be related to increasing both hydrophobic and oleophilic characters of the grafted copolymer after the grafting process.

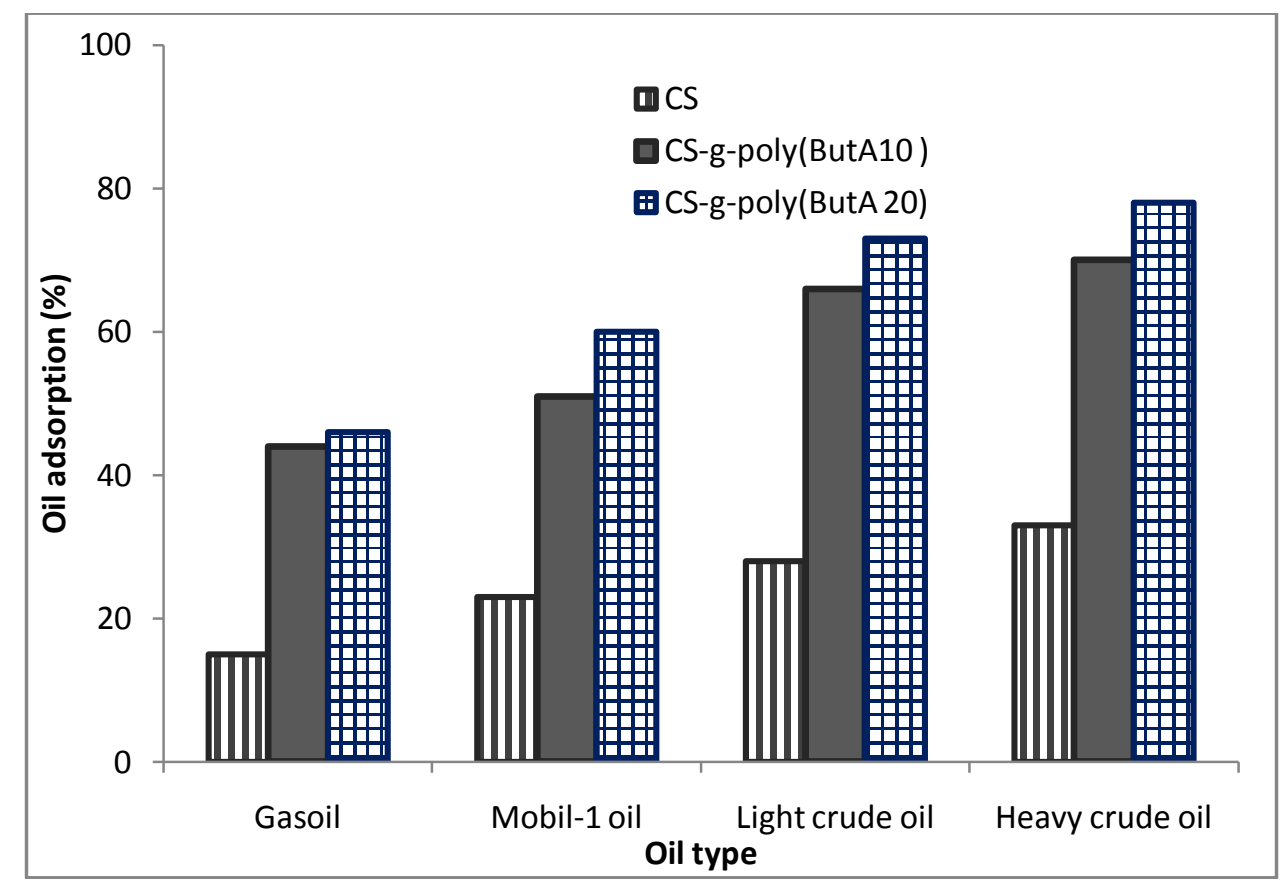

Fig. 9a:- Effect of oil type on the oil adsorption (\%) for CS, CS-g-poly (ButA 10), and CS-g-poly (ButA 20) at constant contact time $(180 \mathrm{~min})$, adsorbent amount $(0.1 \mathrm{~g})$, oil amount $(5 \mathrm{~g})$, agitation rate $(100 \mathrm{rpm})$, and adsorption temperature medium $\left(30^{\circ} \mathrm{C}\right)$.

\section{Effect of initial oil amount:-}

In fact, the variation of the initial amount of oil is very important because it can strongly affect the adsorption kinetics and more specifically the oil adsorbed amount. Fig. $9 \mathrm{~b}$ shows the effect of variation of the initial amount of heavy crude oil from 2.5 to $15 \mathrm{~g}$ on the adsorption process for CS, CS-g-poly (ButA 10), and CS-g-poly (ButA 20).

Generally, adverse effect on the oil adsorption values in all studied samples was noticed with increasing the initial amount of oil up to $15 \mathrm{~g}$. Generally, the oil adsorption (\%) is defined as the ratio of the adsorbed oil amount to the initial amount of oil. Consequently, the oil adsorption values take a negative trend with increasing the initial amount of oil. In addition, the exposed sites in the adsorbent could be saturated with oil molecules, so further increase in the oil amount could be negatively reflect on the adsorption (\%). 


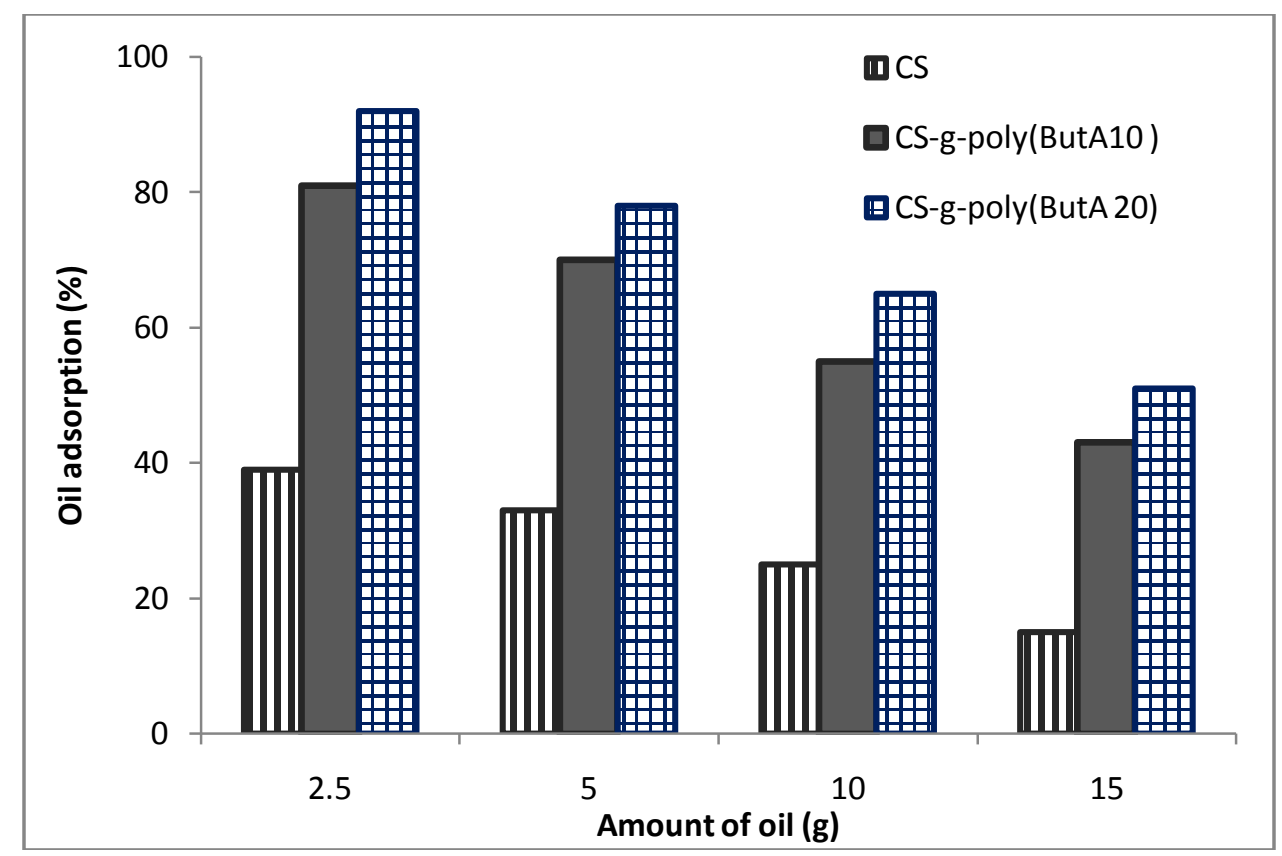

Fig. 9b:- Effect of initial amount of oil on the oil adsorption (\%) for CS, CS-g-poly (ButA 10), and CS-g-poly (ButA 20) at constant contact time (180min), adsorbent amount ( $0.1 \mathrm{~g})$, agitation rate (100 rpm), and adsorption temperature medium $\left(30^{\circ} \mathrm{C}\right)$ using heavy crude oil.

\section{Effect of adsorbent amount:-}

Indeed, the effect of initial amount of adsorbent is an important factor in large scale for the application of adsorbent in the oil spill removal. The consequence of sorbent amount dose on the oil adsorption (\%) was studied in the range from 0.1 to $1 \mathrm{~g}$ as shown in Fig. 9c. It was observed that the oil adsorption (\%) increased gradually with increasing the adsorbent amount from up to 1g. where, maximum maximum values 45, 95, and 100\% for CS, CS-g-poly (ButA 10), and CS-g-poly (ButA 20) were obtained using 1g of adsorbent sample. These results could be attributed to increasing the exposed sites in the prepared samples with increasing the adsorbent dose, which offered for the oil adsorption process per unit gram of sample used (i.e. the higher dose of adsorbents the greater availability of the sorbent sites for the oil spills). Furthermore, the increase in adsorbent amount increases the contact surface of adsorbent particles and it will be more probable for oil spills to be adsorbed. In addition, it was observed that the oil adsorption (\%) for the grafted copolymers was much higher than native chitosan. Where, $5 \mathrm{~g}$ of CS sample was needed to adsorb about $45 \%$ of oil used, while only $0.1 \mathrm{~g}$ of the grafted copolymer can adsorb more than $80 \%$ at the same adsorption conditions, this indicate the increase in the hydrophobic-oleophilic properties after grafting with the butyl acrylate monomer. 


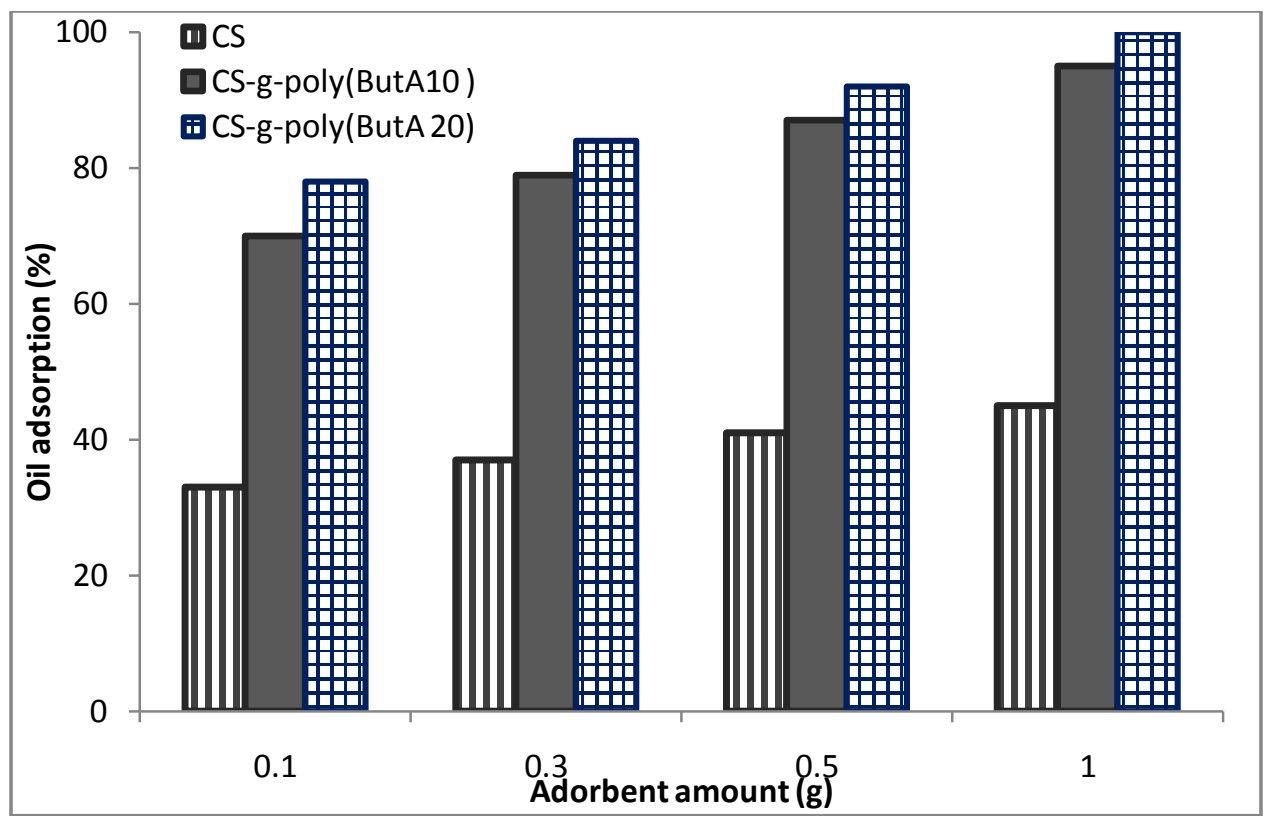

Fig. 9c:- Effect of initial amount of adsorbent on the oil adsorption (\%) for CS, CS-g-poly (ButA 10), and CS-gpoly (ButA 20) at constant contact time (180min), oil amount (5g), agitation rate (100 rpm), and adsorption temperature medium $\left(30^{\circ} \mathrm{C}\right)$ using heavy crude oil.

\section{Effect of contact time:-}

The effect of contact time on the oil adsorption percentage was investigated at different contact time (10-300min) as shown in Fig. 9d. As can be inferred from the obtained results that the oil adsorption (\%) increased exponentially with increasing the contact time up to $180 \mathrm{~min}$, then start to decrease with further increasing of contact time up to $300 \mathrm{~min}$. Also, the results indicate that the adsorption process in case of the grafted copolymer was fast and much higher than chitosan sample. The results revealed that the oil adsorption was fast at the initial stages (up to 180min) of the contact time period, and thereafter it become slower near the equilibrium. This phenomenon was due to the fact that a large number of vacant surface sites were available for adsorption during the initial stage, and after a lapse of time, the remaining vacant surface sites were difficult to be occupied due to repulsive forces between the solute molecules on the solid and bulk phases. On the other hand, with increasing contact time beyond 180min, the affinity of adsorbent towards oil molecules decreased. Besides, desorption process could be occurred which cause decrease in the adsorption percentage 


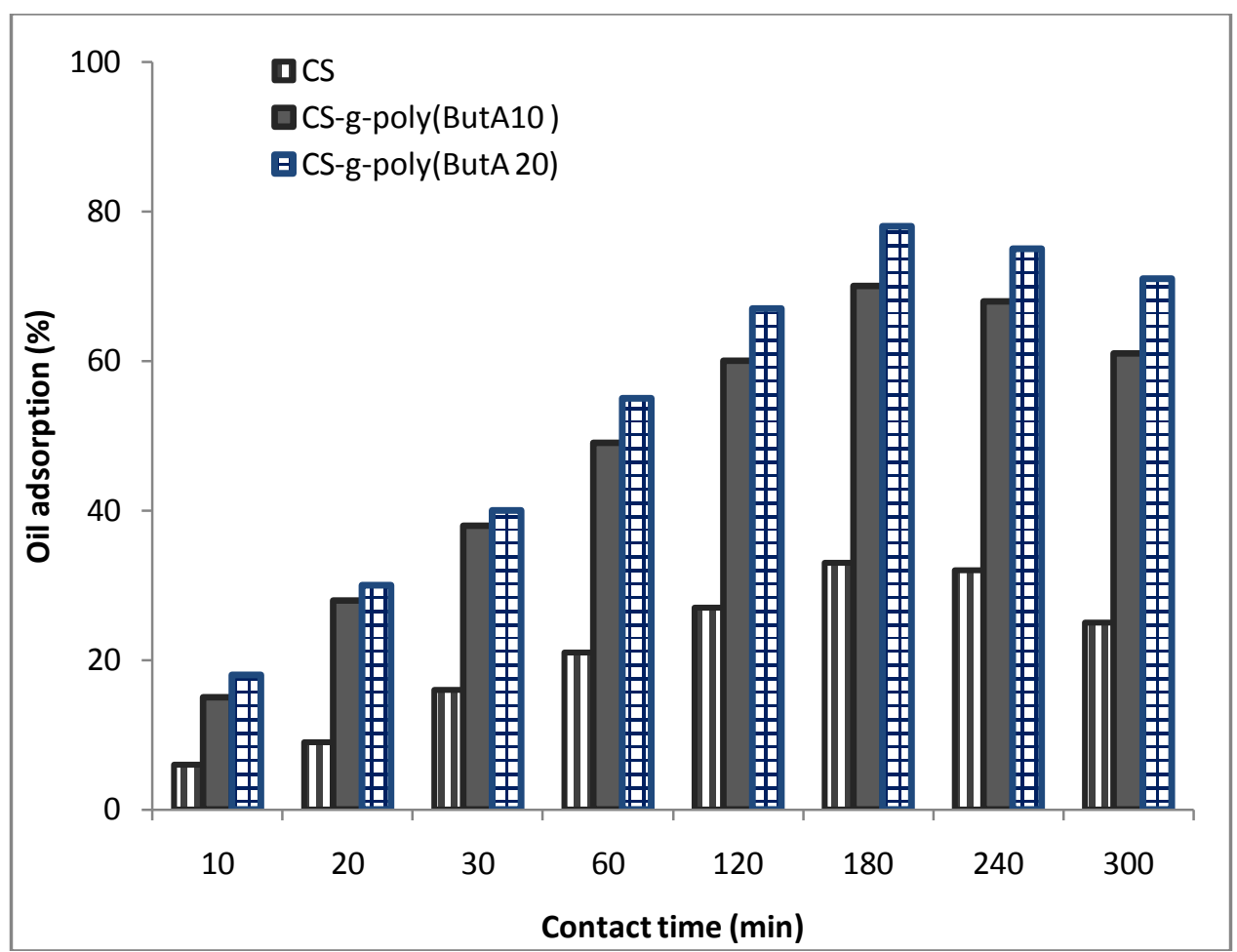

Fig. 9d:- Effect contact time on the oil adsorption (\%) for CS, CS-g-poly (ButA 10), and CS-g-poly (ButA 20), adsorbent amount $(0.1 \mathrm{~g})$, oil amount $(5 \mathrm{~g})$, agitation rate $(100 \mathrm{rpm})$, and adsorption temperature medium $\left(30^{\circ} \mathrm{C}\right)$ using heavy crude oil.

\section{Effect of agitation rate (rpm):-}

Studying the effect of agitation rate on the oil adsorption process is very important to simulate weather and wave conditions in the sea water. Fig. 9e investigates the effect of variation the agitation speed from 50 to $200 \mathrm{rpm}$ on the oil adsorption (\%). The obtained results demonstrate that increasing the agitation rate up to $150 \mathrm{rpm}$ has a positive effect on the oil adsorption (\%) of CS and the grafted copolymers. Where, the oil adsorption increased from 22 to 40\%, from 57 to $90 \%$, and from 66 to $96.5 \%$ in case of CS, CS-g-poly (ButA 10), and CS-g-poly (ButA 20) respectively. In contrast, further increase in the agitation rate up to $200 \mathrm{rpm}$ causes decreasing in the values of oil adsorption (\%). These results could be attributed to that with increasing the agitation rate up to $150 \mathrm{rpm}$ increase the oil dispersion and increase the exposed surface of the adsorbent sample to the oil spills. Additionally, increasing the agitation rate improves the diffusion of oil spills towards the surface of adsorbent samples. On the other hand, the decreasing values of oil adsorption with further increasing of the agitation rate beyond $150 \mathrm{rpm}$ could increase the process of oil-water emulsion, and as a result decrease the attraction forces between the sorbent and oil surface and enhancing the occurrence of oil desorption process. 


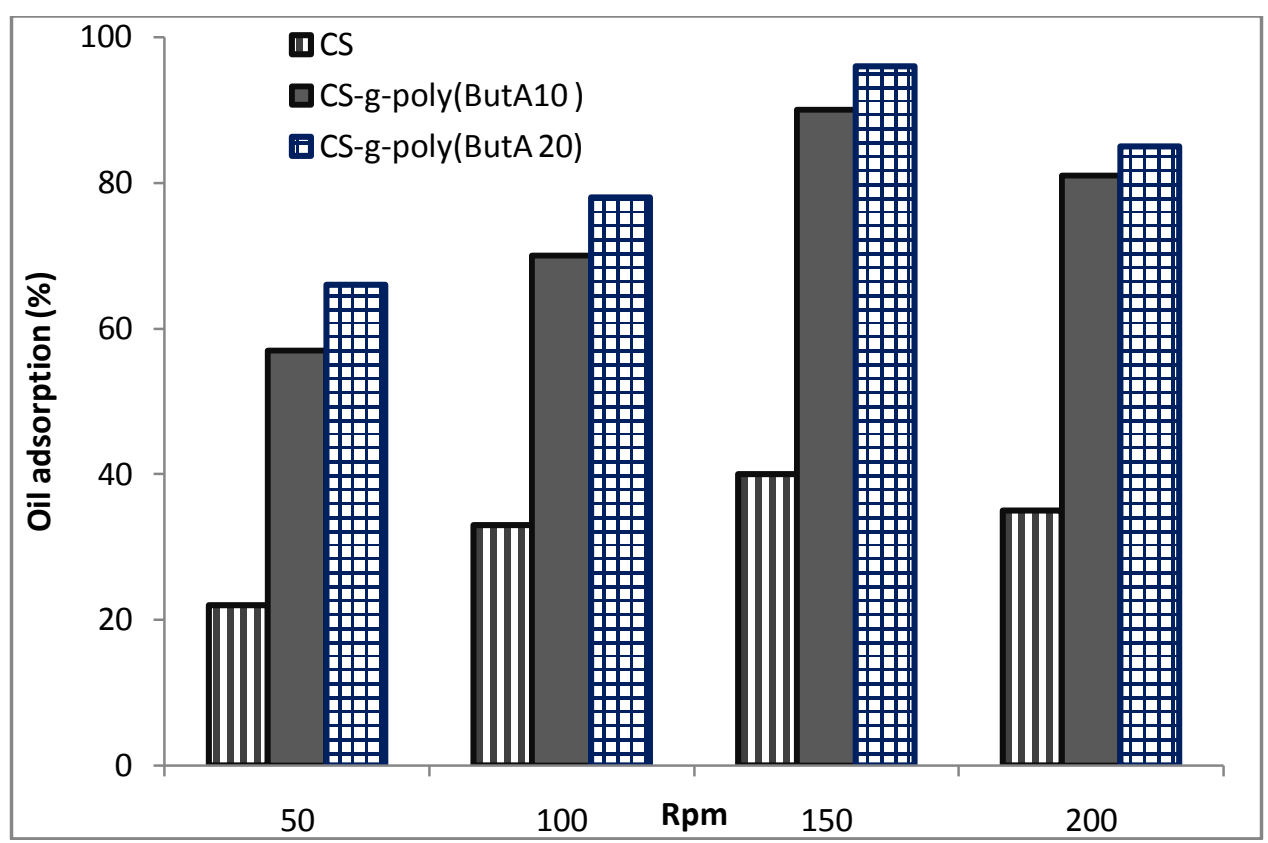

Fig. 9e:- Effect of agitation rate (rpm) on the oil adsorption (\%) for CS, CS-g-poly (ButA 10), and CS-g-poly (ButA 20 ) at constant contact time (180min), adsorbent amount $(0.1 \mathrm{~g})$, oil amount $(5 \mathrm{~g})$, and adsorption temperature medium $\left(30^{\circ} \mathrm{C}\right)$ using heavy crude oil.

\section{Effect of adsorption medium temperature:-}

The behavior of the oil adsorption process for all adsorbent samples were evaluated under different adsorption medium temperatures ranged from 25 to $40^{\circ} \mathrm{C}$ as shown in Fig. 9f. It was comprehensible from results that the oil adsorption (\%) was greatly improved by increasing temperature from 25 to $35^{\circ} \mathrm{C}$, and then tends to decreases with further increasing of temperature up to $40^{\circ} \mathrm{C}$. These results could be explained by increasing the segmental motion for all studied adsorbents, where the diffusion rate of oil spill molecules into the adsorbent surface increased with increasing temperature up to $35^{\circ} \mathrm{C}$. However, increasing temperature beyond $35^{\circ} \mathrm{C}$ (up to $40^{\circ} \mathrm{C}$ ) could increase the speed of Brownian motion of the adsorption medium and more energy is required to adhere the oil molecule onto the adsorbent surface. High temperature causes less probability of oil attachment on the adsorbent surface and the oil desorption process could occur. Moreover, the oil viscosity decrease at higher temperatures and the oil solubility increase, so, the oil adsorption values decreases consequently. 


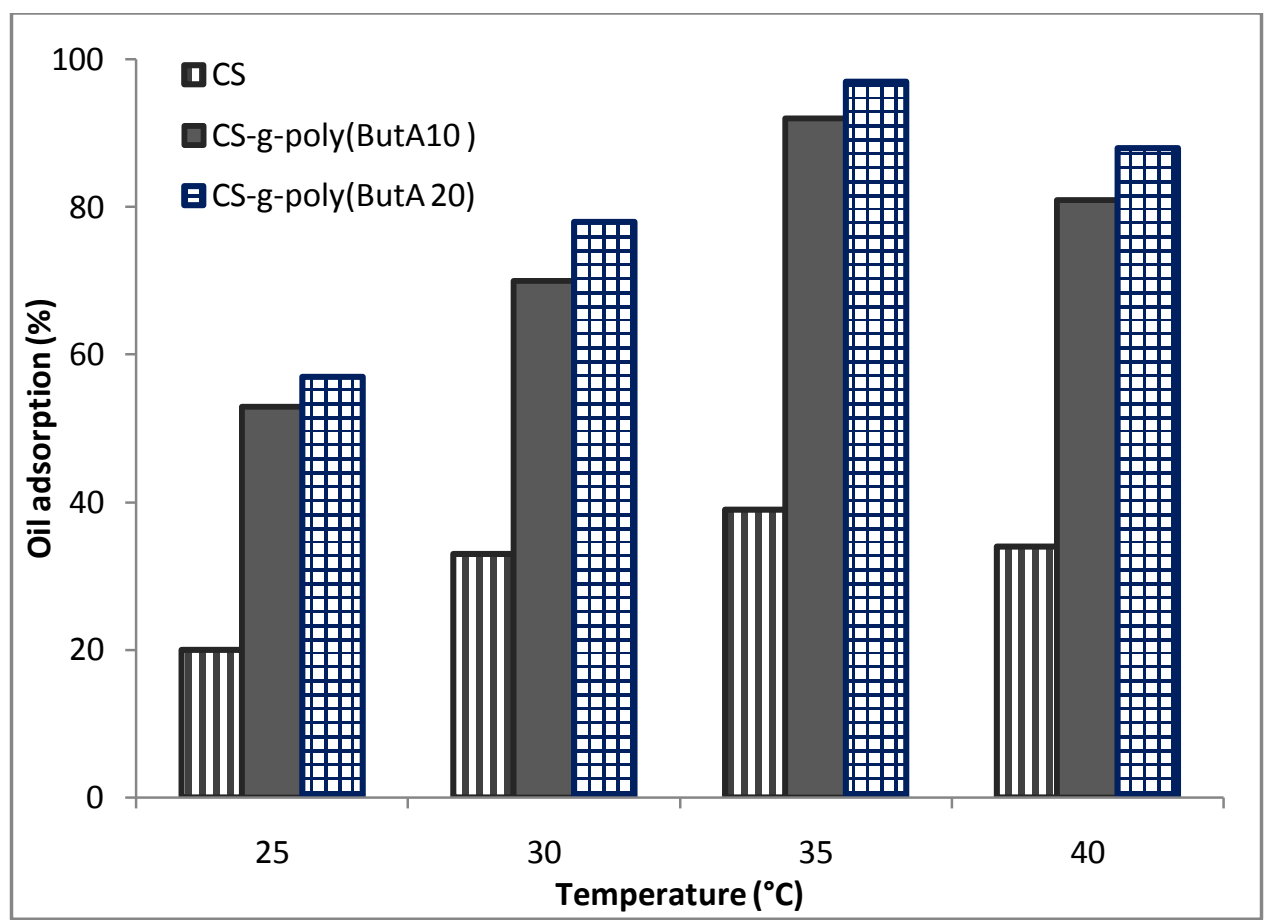

Fig. 9f:- Effect of adsorption medium temperature on the oil adsorption (\%) for CS, CS-g-poly (ButA 10), and CSg-poly (ButA 20) at constant contact time (180min), adsorbent amount $(0.1 \mathrm{~g})$, oil amount $(5 \mathrm{~g})$, and agitation rate (100 rpm) using heavy crude oil.

\section{Conclusion:-}

The hydrophobic-oleophilic characters of low-cost commercial chitosan were greatly improved by grafting of hydrophobic n-butlyl acrylates monomer on its surface using free radical polymerization. The grafted copolymers were characterized using FT-IR, TGA, DSC, and SEM. The influence of chemical grafting of chitosan on the hydrophobic-oleophilic properties was investigated using wettability and liquid uptake experiments. The oil adsorption process was conducted using simulated conditions for sea water. Results indicated that the grafted chitosan-poly (butyl acrylate) copolymer can adsorb heavy crude oil spills more than other oil types. The results of this work provided new hydrophobic-oleophilic adsorbents based on chitosan grafted copolymer for removing heavy crude oil spills from the surface of sea water.

\section{Conflicts of Interest}

The authors have no conflict of interest to declare.

\section{References:-}

1. W. J. Lehr. Review of modeling procedures for oil spill weathering behavior. Advances in Ecological Sciences, 9 (2001) 51-90.

2. X. Cao, G. Yang, S. Wei, H. Han. Sorption of heavy oil onto Jiaozhou Bay sediment. Marine Pollution Bulletin, 62 (2011) 741-746.

3. W. J. Mitsch. The 2010 oil spill in the Gulf of Mexico: What would Mother Nature do?, Ecol. Eng., 36 (2010) $1607-$ 1610.

4. A.L. Ahmad, S. Sumathi, B.H. Hameed. Adsorption of residue oil from palm oil mill effluent using powder and flake chitosan: equilibrium and kinetic studies, Water Res., 39 (2005) 2483-2494.

5. J. M. Nori, B. N. S. Anurag, V. A. K. Thimmarusu. An experimental study on the absorption capacity and reusability of cotton and the effect of salinity on crude oil fractions on sea water (Bay of Bengal). International Journal of Research in Engineering and Technology, 3 (2014) 210-216.

6. S. Xin, Q. Li, W. Zhang, Y. Wan. Synthesis and Absorbency of Oil / Organics by a Novel Modified $\mathrm{SiO}_{2}-\mathrm{Acrylate}$ Composite Resin. Journal of Materials and Applications, 1 (2014) 33-43.

7. B. Tansel, B. Pascual. Removal of emulsified fuel oils from brackish and pond water by dissolved air flotation with and without polyelectrolyte use: pilot-scale investigation for estuarine and near shore applications. Chemosphere, 85 (2011) 1182-1186. 
8. M. Inagaki, A. Kawahara, Y. Nishi, N. Iwashita. Heavy oil sorption and recovery by using carbon fiber felts. Carbon, 40 (2002) 1487-1492.

9. M. O. Adebajo, R. L. Frost, J. T. Kloprogge, O. Carmody, S. Kokot. Porous materials for oil spill cleanup, A Review of Synthesis. J. porous. Mater., 10 (2003)159-170.

10. S. Panpanit, C. Visvanathan. The role of bentonite addition in UF flux enhancement mechanisms for oil/water emulsion. J. Membr. Sci., 184 (2001) 59-68.

11. H. Zhu, S. Qiu, W. Jiang, D. Wu, C. Zhang. Evaluation of electrospun polyvinyl chloride/polystyrene fibers as sorbent materials for oil spill cleanup. Environ. Sci. Technol., 45 (2011) 4527-4531.

12. S. M. Sidik, A. A. Jalil, S. Triwahyono, S. H. Adam, M. A. H. Satar, B. H. Hameed. Modified oil palm leaves adsorbent with enhanced hydrophobicity for crude oil removal. Chem. Eng. J., 203 (2012) 9-18.

13. M. Saito, N. Ishii, S. Ogura, S. Maemura, H. Suzuki, Development and water tank tests of Sugi bark sorbent (SBS). Spill. Sci,. Technol. B. 8 (2003) 475-482.

14. M. S. Mohy Eldin, A. M. Omer, M. A. Wassel, T. M. Tamer, M. S. Abd Elmonem, S. A.Ibrahim. Novel smart pH sensitive chitosan grafted alginate hydrogel microcapsules for oral protein delivery: I. preparation and characterization. Int. J. Pharm. Pharm. Sci., 7 (2015) 320-326.

15. M. Rinaudo. Chitin and chitosan: properties and applications. Prog. Polym. Sci., 31 (2006) 603-32.

16. N. A. Abdul Aziz, A. Abu Bakar, A. Hassan, N. Azmi, Synthesis of chaitozan-grafted-poly(methyl methacrylate) with fenton's reagent $\left(\mathrm{Fe}_{2}{ }^{+}-\mathrm{H}_{2} \mathrm{O}_{2}\right)$ as a redox initiator, Malays. J. Analyt. Sci. 18 (2014) 415 - 422.

17. M. S. Mohy Eldin, E. A. Soliman, A. I., Hashem, T. M. Tamer. Antibacterial activity of chitosan chemically modified with new technique. Trends in Biomaterilas \& Artificial Organs, 22, (2008) 121-133.

18. M. S. Mohy Eldin, E. A. Soliman, A. I., Hashem, T. M. Tamer. Antimicrobial activity of novel aminated chitosan derivatives for biomedical applications. Advances in Polytechnic, 31, (2012) 414-428.

19. M. S. Mohy Eldin, E. A. Soliman, A. I., Hashem, T. M. Tamer, M.M Sabet. Antifungal activity of aminated chitosan against three different fungi species. Key Engineering Materials-Current State-of-the-Art on Novel Materials; Vol 1, Chapter 26, (2013) 515-431.

20. M. S. Mohy Eldin, A. M. Omer, A. I., Hashem, T. M. Tamer. Preparation, characterization and antimicrobial evaluation of novel cinnamyl chitosan Schiff base. International Journal of Advanced Research, 3, (2015a) $741-755$.

21. M. S. Mohy Eldin, A. M. Omer, A. I., Hashem, T. M. Tamer.. Wound dressing membranes based on chitosan: Preparation, characterization and biomedical evaluation. International Journal of Advanced Research, 3(8), (2015) 908-922.

22. M. S. Mohy Eldin, T. M. Tamer, M. A. Abu Saied, E. A. Soliman, N. K. Madi, I Raga, I. Fadel. Click grafting of chitosan onto PVC surfaces for biomedical applications. Advances in Polymer Technology, (2015) 1-12.

23. M.S. Mohy Eldin, A. M. Omer, M.A. Wassel, T.M. Tamer, M.S. Abd-Elmonem, S.A. Ibrahim. Novel smart pH sensitive chitosan grafted alginate hydrogel microcapsules for oral protein delivery: I. Preparation and characterisation. Journal of Applied Pharmaceutical Science, 7(10), (2015) 320-326.

24. M.S. Mohy Eldin, A. M. Omer, M.A. Wassel, T.M. Tamer, M.S. Abd-Elmonem, S.A. Ibrahim. Novel smart pH sensitive chitosan grafted alginate hydrogel microcapsules for oral protein delivery: II. Evaluation of the swelling behavior. Journal of Applied Pharmaceutical Science, 7(10), (2015) 331-337.

25. E. Kenawy, F.I. Abdel-Hay, M.S. Mohy Eldin, T.M. Tamer, E.M.A. Ibrahim. Novel aminated chitosan-aromatic aldehydes Schiff bases: Synthesis, characterization and bio-evaluation. International Journal of Advanced Research, 3(2), (2015) 3563-572.

26. T.M. Tamer, K. Valachová, M.S. Mohy Eldin, L. Šoltés. Free radical scavenger activity of chitosan and its aminated derivative. Journal of Applied Pharmaceutical Science, 6(4), (2016)195-201.

27. T.M. Tamer, K. Valachová, M.S. Mohy Eldin, L. Šoltés. Scavenger activity of cinnamyl chitosan Schiff base. Journal of Applied Pharmaceutical Science, 6(1), (2016) 130-136.

28. K. Valachová, T.M. Tamer, M.S. Mohy Eldin, L. Šoltés. Radical-scavenging activity of glutathione, chitin derivatives and their combination. Chemical Papers, 70(6), (2016) 820-827.

29. M. A. L. Milhome, D. Keukeleire, J. P. Ribeiro, R. F. Nascimento. Removal of phenol and conventional pollutants from aqueous effluent by chitosan and chitin. Quim. Nova, 32 (2009) 2122-2127.

30. O. G.Valdez, R. C.Hartley, E. S. Guerra, P. Champagne, M. F. Cunningham. Modification of chitosan with polystyrene and poly (n-butyl acrylate) via nitroxide-mediated polymerization and grafting from approach in homogeneous media. Polym. Chem., 6 (2015) 2827-2836.

31. K.V.H. Prashanth, R.N. Tharanathan. Studies on graft copolymerization of chitosan with synthetic monomers. Carbohydrate Polymers, 54 (2003) 343-351.

32. D. W. Jenkins, S. M. Hudson. "Review of Vinyl Graft Copolymerization Featuring Recent Advances toward Controlled Radical-Based Reactions and Illustrated with Chitin/Chitosan. Trunk Polymers," Chemical Reviews, 101 (2001) 3245-3273. 
33. H.H. Sokker, Naeem M. El-Sawy, M.A. Hassan, Bahgat E. El-Anadouli. Adsorption of crude oil from aqueous solution by hydrogel of chitosan based polyacrylamide prepared by radiation induced graft polymerization. Journal of Hazardous Materials, 190 (2011) 359-365.

34. R. Nithya, P. N. Sudha. Grafting of n-butyl acrylate on to chitosan by ceric ion initiation and its antimicrobial activity. Der Pharmacia Lettre, 6 (2014) 58-64.

35. B. O. Jung, C. H. Kim, K. S. Choi, Y. M. Lee, J. J. Kim. "Results, Phosphorous Containing Chitosan Beads for Controlled Oral Drug. Journal of Applied Polymer Science, 72 (1999) 1713-1719.

36. F. Kotze, H.L. Lueßen, B.J. De Leeuw, A.G. De Boer, J.C. Verhoef; H.E. Junginger. N-trimethyl chitosan chloride as a potential absorption enhancer across mucosal surfaces: in vitro evaluation in intestinal epithelial cells (Caco2).Pharmaceutical Research, 14 (1997) 1197-1202.

37. V.K. Mourya, N.N. Inamdar. Chitosan-modifications and applications: Opportunities galore. React Funct Polym., 68 (2008) 1013-1051.

38. R. Jayakumar, M. Prabaharan, R. L. Reis and J. F. Mano. Graft copolymerized chitosan-present status and applications.Carbohydr. Polym., 62 (2005) 142-158.

39. J. Retuert, M. Pedram.Cocatalyst effect in potassium persulfate initiated grafting onto chitosan. Polym. Bull., 31(1993) 559-562.

40. S. Yoshikawa, T. Takayama. N. Tsubokawa. Grafting reaction of living polymer cations with amino groups on chitosan powder. J. Appl.Polym. Sci., 68 (1998) 1883-1889.

41. M. Pati, P.L. Nayak. Graft copolymerization of methyl acrylate on chitosan: Initiated by ceric ammonium nitrate as the initiator-characterization and antimicrobial activity. Advances in Applied Science Research, 3 (2012) 1646-1654.

42. T. M. Tamer, A. M. Omer, M. A. Hassan, M. E. Hassan, M. M. Sabet, M. S. Mohy Eldin. Development of thermosensitive poly $\mathrm{N}$-isopropyl acrylamide grafted chitosan derivatives. Journal of Applied Pharmaceutical Science, 5 (2015) 001-006.

43. Z. Yang, H. Yang, Z. Jiang, T. Cai, H. Li, H. Li, A. Li. R. Cheng. Flocculation of both anionic and cationic dyes in aqueous solutions by the amphoteric grafting flocculant carboxymethyl chitosan-graft-polyacrylamide. Journal of Hazardous Materials, 254 (2013) 36-45.

44. M. M. Islama, S. M. Masumb, M. M. Rahmana, M. A. I. Mollab, A. A. Shaikhc, S. K. Roya. Preparation of chitosan from shrimp shell and investigation of its properties, Int. J. Basic App. Sci. IJBAS-IJENS., 11 (2011) 77- 80.

45. G. Rigby. Substantially undegraded deacetylated chitin and processes for producing the same, patent. USA., 2, 040, 879 (1936).

46. M. S. Mohy Eldin, H. M. El-Sherif, E. A. Soliman, A. A. Elzatahry, A. M. Omer. Polyacrylamide-grafted carboxymethyl cellulose: smart pH-Sensitive hydrogel for protein concentration. J. Appl. Polym. Sci., 12 (2011) 46979.

47. M. S. Mohy Eldin, A. M. Omer, E. A. Soliman, E. A. Hassan. Superabsorbent polyacrylamide grafted carboxymethyl cellulose pH sensitive hydrogel: I. Preparation and characterization. Desalin. Water Treat., 51 (2013) 3196-206.

48. B. Alireza, T. Jun, M. Gordon.Standardization of oil sorbent performance testing, J. Test. Eval., 43 (2015) 1- 8.

49. A. Nikkhah, H. Zilouei, A. Asadinezhad, A. Keshavarz. Removal of oil from water using polyurethane foam modified with nanoclay, Chem. Eng. J., 262 (2015) 278-285.

50. M. Patowary, R. Ananthakrishnan, K. Pathak. Superhydrophobic and oleophilic barium sulfate material for oil spill clean-ups: Fabrication of surface modified sorbent by a one-step interaction approach, J. Environ. Chem. Eng., 2 (2014) 2078-2084.

51. Pawlak, M. Mucha. Thermogravimetric and FTIR studies of chitosan blends. Thermochim Acta. 396(2003) $153-166$.

52. J. Zawadzki, H. Kaczmarek. Thermal treatment of chitosan in various conditions, Carbohydr. Polym., 80 (2010) 394400.

53. J. L. Fuente, M. F. Garcia, E. L. Madruga. Characterization and Thermal Properties of Poly(n-butyl acrylate-gstyrene) Graft Copolymers. Journal of Applied Polymer Science, 80 (2001)783-789.

54. F. C. d. F. Barrosa, L. C. G. Vasconcellosb, T. V. Carvalhoc, R. F. d. Nascimentoa, Removal of petroleum spill in water by chitin and chitosan, Orbital: Electron. J. Chem., 6 (2014) 70-74. 